\title{
Militärsanitätswesen.
}

\section{Fortschritte auf dem Gebiet des Militẳrsanitätswesens.}

\section{Von Generaloberarzt Dr. E. Schill in Dresden.}

Im nachstehenden soll ein kurzer Ueberblick über die Leistungen und Fortschritte auf dem Gebiet des Militärsanitätswesens der letzten $J$ ahre in der deutschen Armee gegeben werden.

Für die persönliche Stellung der Sanitätsoffiziere des Deutschen Heeres von höchster Wichtigkeit ist die "Allerhöchste Verordnung" über die Ehrengerichte der Sanitätsoffiziere vom 9. April 1902, welche der gleichen Verordnung für die Offiziere durchweg entspricht. Als weiterer wichtiger Fortschritt war im gleichen Jahre zu begrüßen die Schaffung des "Wissenschaftlichen Senats bei der Kaiser Wilhelms-Akademie", ferner die Re. gelung der militärärztlichen Fortbildungskurse, von denen in Berlin jährlich ein dreiwöchentlicher für Oberstabs- bis Assistenzärzte, ein vierwöchentlicher hygienischer für Ober- und Assistenzärzte und ein vierwöchentlicher zur Ausbildung in chemischen und bakteriologischen Untersuchungen und an den Universitäten dreiwöchentliche Kurse für Sanitätsoffiziere des aktiven Dienst- und des Beurlaubtenstandes abgehalten werden.

Als bedeutsame Errungenschalt des letzten Jahres darf die Schaffung von Stellen für Sanitätsinspekteure (Generalärzte mit Generalmajorsrang) - wenigstens in der preußischen, leider aber noch nicht in der bayerischen und säehsischen Armee - hier nicht übergangen werden. Die Sanitätsinspekteure sind berufen, im Krieg und Frieden ein wichtiges Zwischenglied zwischen dem Generalstabsarzt und den Korpsgeneralärzten zu bilden.

Ias wichtigste organisatorische Novum ist die lange sehnlich erwartete neue „Kriegssanitätsordnung", welche am 27. Januar d. J. von Sr. Majestät dem Kaiser genehmigt wurde und die gleiche Dienstvorschrift vom 10. Januar 1878 zu ersetzen bestimmt ist. Ihre Würdigung wird in dieser Wochenschrift später erfolgen.

Auf dem Gebiet der Organisation des Sanitätsdienstes haben sich im übrigen zwar keine grundsätzlichen Neuerungen vollzogen, doch ist mehr als eine bemerkeńswerte Verbesserung zu verzeichnen. Als das ostasiatische Expeditionskorps 1900 auszog, galt es auch den Sanitätsdienst den neuen eigenartigen Verhältnissen anzupassen, unter den sich freiwillig zur Teilnahme Meldenden kritisch Auswahl zu halten, sie durch eine übersichtliche, leicht verständliche Vorschrift über zweckmäßiges Verhalten während des Seetransportes und in China zu belehren, für eine praktische Ausrüstung der Truppe mit geeigneter Kleidung und Nahrung, vor allem aber für Versorgung mit einwandfreiem Trinkwasser Vorkehrungen zu treffen und die Sanitätsformationen mit geeignetem Material, darunter auch Trinkwassersterilisations- und Dampf-, sowie Formalin-Desinfektionsapparaten und Röntgeneinrichtungen auszustatten. Aehnliche, aber umfassendere Aufgaben wurden dem Sanitätsdienst gestellt, als der Aufstand in Südwestafrika immer weiteren Umfang annahm und insbesondere Typhuserkrankungen in größerer Zahl auftraten. Dort galt es inmitten wüster Landstriche Lazarette einzurichten und der fortschreitenden Erkrankungen Herr zu werden. Zum erstenmal wurden in der deutschen Armee Schutzimpfungen gegen Typhus vorgenommen. Wenn die Zahl derselben auch eine beschränkte geblieben ist; so steht doch zu hoffen, daß wir aus ihnen erkennen können, ob wir - wenn auch nur für bestimmte militärische Verhältnisse - Schutzimpfungen gegen andere Krankheiten als gegen Pocken künftighin als 
prophylaktische Maßregel in Aussicht zu nehmen haben. Das Jahr 1901 brachte als Novum die Einrichtung von Beobachtungslazaretten auf den Truppenübungsplätzen, nach welchen die aus Ostasien zurückbeförderten Teile des Expeditionskorps einer gesundheitlichen Priifung unterzogen wurden, um Verschleppung von Typhus und Ruhr nach Deutschland zu verhüten.

Bei zahlreichen Verbesserungen bestehender Einrichtungen waren Sanitätsoffiziere berufen mitzuwirken: bei Neubau und Umbau von Kasernen, Lagern und Lazaretten, der Ernährung der Mannschaften u. dgl. Als besonders wichtig sind hier zu nennen die „Allgemeinen Grundsätze für den Neubau von Gar. nisonlazaretten " 1904, die Bestimmungen über Einrichtung von Röntgeninstituten und Zahnstationen, die bessere Ausstattung der Revierkrankenstuben, die Schaffung von weiteren Militärgenesungsheimen (beim Garde-, XIIL, XIV., XIX. Korps und in Bayern), Eröffnung des Genesungsheims für Offiziere in Arco in Tirol 1902, die Bestimmungen über Badekuren 1902 und später 1905 (Kurvorschrift), die Herausgabe der „Belehrung über Hitzschlag auf Märschen vom 2. Juni 1904" und die Bestimmung, daß Offiziere und Mannschaften über das Wesen und die Verhütung venerischer Krankheiten zu belehren sind (1904). - In Kaserne n wurden an vielen Orten Verbesserungen getroffen in bezug auf Beköstigung, Trinkwasserversorgung durch Anschluß an bestehende städtische Wasserleitungen, durch Anlegung eigener Leitungen oder Sanicrung der Brunnenanlagen, Trennung der Wohn-, von den Schlaf-, Putz- und Waschräumen, Neuanlage und Umban von Aborten und Pißanstalten, Einrichtung von Massenbädern 11. a. m. Lazarettneubauten zeigen meist alle Errungenschaften der Neuzeit in bezug auf hygienische Einrichtungen. Auch eine große Zahl älterer Lazarette, die Mängel und Unvollkommenheiten zeigten, haben Verbesserungen erfahren: Neubau von Operationssälen mit allen Einrichtungen für aseptische Wundbehandlung, Einrichtung neuer spezialistischer Stationen, Bau von Sonderhäusern für ansteckende Kranke, Desinfektionsanlagen und dergleichen.

Auch für die Sanitßatsausrïstungen fïr den Krieg und Frieden haben die letzten Jahre Fortschritte gebracht. 1890 wurden iiber 3 Millionen Mark für Neubeschaffungen von Instrumenten zur Aseptik bei Feldformationen, Tablettenausrüstung, Sterilisationsund Röntgenapparate und komprimierte Verbandstoffe bewilligt und zahlreiche Verbesserungen am Sanitätsmaterial durchgefiihrt. Erwähnt sei hier noch die Einführung eines tragbaren bakteriologischen Laboratoriums für das Feld und eines neuen „V erbandpäckchens", welches dem Verwundeten oder seinem Helfer" ermöglicht, den Verband anzulegen, ohne die mit der Wunde in Berührung kommenden Kompressen anzufassen.

Von der unermüdlichen wissenschaftlichen Tatigkeit auf dem Gebiet des Militärsanitätswe sens legen Zeugnis ab: die von der Medizinalabteilung des Königlich Preußischen Kriegsministeriums bearbeiteten "Sanitätsberichte", welche ein hocherfreuliches stetiges Herabgehen der Mortalität und Morbidität nachweisen, die von derselben Dienststelle herausgegebenen "Veröffentlichungen aus dem Gebiet des Militärsanitätswesens", welche besonders wichtige Fragen des Sanitätsdienstes behandeln, z. B. Trinkwasserversorgung, Schutzimpfung gegen Typhus, Verhütung von Tetanus nach Platzpatronenverletzungen, plötzliche Todesfälle, Schußverletzungen, Bekämpfung des Typhus und der Dysenterie, Beurteilung der Herzkrankheiten, Feststellung regelwidriger Geisteszustände, weiterhin die "Bibliot hek von Coler", herausgegeben von Schjerning, von der bereits 24 Bände erschienen sind, welche u. a. behandeln Pocken, Diphtherie, innere Krankheiten, Pest, Cerebrospinalmeningitis als Heeresseuchen, Infektionskrankheiten, deren experimentelle Diagnostik, Serumtherapie und Prophylaxe, Hitzschlag auf Märschen, Physiologie des Marsches, Kriegsepidemien, Verwundungen durch moderne Kriegswaffen, Grundsätze für den Bau von Krankenhäusern, Operations- und Verbandtechnik, Invalidenversorgung und Begutachtung. Die Entsendung von Sanitätsoffizieren auf den russisch-japanischen Kriegsschauplatz ermöglichte Einblicke in die Schwierigkeiten der sanitären Fürsorge be Riesenheeren, welche fern von ihrem Heimatlande sich mit den vervollkommensten Waffen der Neuzeit bekämpfen.

In den militärärztlicheu Vereinigungen innerhalb der einzelnen Korps entfaltete sich rege wissenschaftliche Tätigkeit; als Publikationsorgan für wissenschaftliche Fachfragen diente die von R. v. Leuthold begründete "Deutsche militärärztliche Zeitschrift", welche seit Beginn des laufenden Jahres zweimal monatlich erscheint. Von selbständigen Werken, welche ausschließliche oder vorwiegende militärärztliche Themata behandeln, seien aus den letzten Jahren noch besonders hervorgehoben: Mit dem Beginn des Jahrhunderts fand das Handbuch der Militärkrankheiten von Düms seinen AbschluB. Mit Militärhygiene befaßten sich eine Reihe Autoren, so Hille r (Gesundheitspflege des Heeres 1905), Kulcke Grundzüge der Militärgesundheitspflege 1903), B a r th el m es (Grund- züge der Gesundheitspflege für Truppen-Offiziere 1907), Schöfer (Leitfaden der Militärhygiene 1900), Croner (Gesundheitsbüchlein des Soldaten 1904). Innere "Armeekrankheiten" behandelten außer den Verfassern der vorerwähnten Bände der „Veröffentlichungen aus dem Gebiete des Militärsanitätswesens" und der "Bibliothek von Coler" in selbständigen Werken: Knaak (Krankheiten im Kriege 1900), Hiller (Hitzschlag auf Märschen 1902), Kamen (Die Infektionskrankheiten mit besonderer Berücksichtigung der Armeeverhältnisse 1905), Bon ette (Der Hitzschlag in der Armee 1905). Militärchirurgische Werke veröffentlichten Schjerning, Thöle und Voss (Die Schußverletzungen 1902), Kranzfelder und Schwiening (Funkenphotographie zur Darstellung der Geschoßwirkung im menschlichen Körper 1903), Seydel (Lehrbuch der Kriegschirurgie, 2. Auflage, 1905), H. Fischer (Leitfaden der kriegschirurgischen Operations- und Verbandtechnik 1905), Rotter (Die typischen Operationen, 7. Auflage, 1905).

Die kriegschirurgischen Beobachtungen und Erfahrungen aus dem Burenkrieg. dem Krieg gegen China, dem russisch-japanischen Krieg und dem südwestafrikanischen Aufstand, finden sich fast nur in Zeitschriften zerstreut; zusammenfassende Besprechung fanden sie in dem allbekannten Rothschen Jahresbericht und in dem Abschnitt „Kriegschirurgie “ in dem Jahresbericht über die Leistungen und Fortschritte der gesamten Medizin (früher Virchow-Hirsch), bearbeitet von Schjerning.

Ein Gebiet, welches erst im begonnenen Jahrhundert ausgebaut worden ist, ist die Sanitätstaktik. Außer Sanitätsoffizieren waren hier Offiziere, meist vom Generalstab, tätig, so v. Kries (Sanitätsdienst vor dem Feinde 1901), v. Oven (Taktische Ansbildung des Sanitätsoffiziers 1901), Hausenblas (Sanitätsdienst in vorderster Linie bei den drei Hauptwaffen 1902), Löffler (Sanitätstaktik 1902), Etzel (Die Befehlsgebung der Sanitätsoffiziere im Felde 1904). Von Sanitätsoffizieren rühren Arbeiten über Sanitätstaktik her, 11. a. von Timann (Der Sanitätsdienst auf dem Schlachtfeld 1901), Dautwitz (Sanitätstaktische Ausbildung der Sanitätsoffiziere 1901), Cron (Beziehungen des Feldsanitätsdienstes zum Felddienst 1901).

Als Grundlagen für den Unterricht des Sanitätspersonals dient das 1902 erschienene „Unterrichtsbuch für Sanitätsmannschaften "; von besonderem Wert für die Ausbildung der Kräfte der freiwilligen Hilfe aber sind: Rupprecht, Krankenpflege im Krieg und Frieden (1901), Rühlemann, Unterrichtsbuch für die Sanitätskolonnen des Roten Kreuzes (15. Auflage, 1905) und Hald er Cramer, Grundril3 der freiwilligen Krankenpflege im Anschluß an den Sanitätsdienst (1905)

Ein Werk, welches die verschiedensten Seiten des Militärsanitätswesens im Kriege berührt, ist das 1902 erschienene Buch Aerztliche Kriegswissenschaft, Vierzehn Vorträge", mit Beiträgen von R. Koch (Seuchenbekämpfung im Kriege), M. Kirchner (Ernahrung und Trinkwasserversorgung), Schumburg (Hygiene des Marsches, Unterkunft), Krocker (Bekleidung und Ausrüstung) v. Bergmann (Erste Hilfe auf dem Schlachtfeld, A- und Antisepsis, Schußwunden des behaarten Kopfes), Kuttner (Schußwunden der Extremitäten), Kö nig (Schußverletzungen des Rumpfes) Köhler (Hieb- und Stichwunden), Schjerning (Organisation des Sanitätsdienstes im Felde), Werner (Krankentransport und -unterkunft im Kriege), Schaper (Krankenpflege im Kriege) und Kü bler (Kriegssanitätsstatistik).

Auf dem Gebiet der Feststellung von Dienstbeschadigungen waren wesentliche Neuerungen die "Pensionierungsvorschrift vom 2. Juni 1900" und das "Offizierspensions- und Mann. schaftsversorgungsgesetz vom 31. Mai 1906 ", welche mannigfache Aenderungen der "Dienstanweisung zur Beurteilung der Militärdienstfähigkeit und zur Ausstellung von militärärztlichen Zeugnissen vom 13. Oktober 1904" zur Folge hatten. Einen vorzüglichen Kommentar dazu schrieb Paalzow (Invaliden-Versorgung und -Begutachtung, Berlin, A. Hirschwald, 1906). 